\title{
Automated and Weighted Self-Organizing Time Maps
}

\author{
Peter Sarlin ${ }^{\mathrm{a}, \mathrm{b}, *}$ \\ ${ }^{a}$ Center of Excellence SAFE at Goethe University, Frankfurt, Germany \\ ${ }^{b}$ RiskLab at IAMSR, Åbo Akademi University (Turku) and Arcada University of Applied \\ Sciences (Helsinki), Finland
}

\begin{abstract}
This paper proposes schemes for automated and weighted Self-Organizing Time Maps (SOTMs). The SOTM provides means for a visual approach to evolutionary clustering, which aims at producing a sequence of clustering solutions. This task we denote as visual dynamic clustering. The implication of an automated SOTM is not only a data-driven parametrization of the SOTM, but also the feature of adjusting the training to the characteristics of the data at each time step. The aim of the weighted SOTM is to improve learning from more trustworthy or important data with an instance-varying weight. The schemes for automated and weighted SOTMs are illustrated on two real-world datasets: (i) country-level risk indicators to measure the evolution of global imbalances, and (ii) credit applicant data to measure the evolution of firm-level credit risks.
\end{abstract}

Keywords: Self-Organizing Time Map, weighting schemes, quality measures

\section{Introduction}

This paper proposes schemes for automated and weighted Self-Organizing Time Maps (SOTMs). The SOTM [16] is a recently introduced adaptation of Kohonen's 1982 Self-Organizing Map (SOM) that enables visual dynamic clustering. The SOTM provides means for a visual approach to so-called evolutionary clustering (EC) [2], which aims at processing temporal data by producing a sequence of clustering solutions. Chakrabarti et al. 2] describe the aim of EC to be a balance between faithfulness of the clustering to current data and comparability with the previous clustering result, and relate it to four virtues: $(i)$ consistency (i.e., familiarity with the previous clustering), (ii) noise removal (i.e., a historical consistent clustering increases robustness), (iii) smoothing (i.e., a smooth view of transitions), and ( $i v$ ) cluster correspondence (i.e., relation to historical context). Recent research has also provided other

${ }^{*}$ Corresponding author: RiskLab at IAMSR, Department of Information Technologies, Åbo Akademi University. Postal address: Joukahaisenkatu 3-5, 20520 Turku, Finland. Email: psarlin@abo.fi. Tel: +35822154670. 
approaches to adaptive clustering, but with different inspiration. Some examples constitute of kernel spectral clustering with memory [12 and incremental $k$-means [3]. Beyond only performing EC, the SOTM is a visual approach, as it provides means for a low-dimensional representation of the high-dimensional data. The SOTM is essentially a sequence of one-dimensional SOMs on data ordered in consequent time units and represents both time and data topology on a two dimensional grid of reference vectors. Hence, the topology preservation of the SOTM may preserve data topology on the vertical direction and time topology on the horizontal direction.

The SOTM is applicable in a wide range of application areas with data along three dimensions of a data cube: (i) cross-section, (ii) time, and (iii) inputs. This is a common setting in many domains, such as social sciences, finance and engineering, with data for several entities, reference periods and explanatory variables. Since the introduction of the approach, the SOTM has been combined with hierarchical clustering methods to better reveal emerging, changing and disappearing clusters [18 and shown to not be restricted to illustrating changes over the time, but equally applicable for abstractions of changes in cluster structures over any variable of ordinal, cardinal or higher level of measurement [15]. The SOTM has also been applied to a range of tasks, such as visual explorations of cross-sectional changes in welfare and poverty [16], temporal customer segmentation [20], temporal customer behavior analysis [21], exploring and comparing green vs. non-green customer segmentations [4, an abstraction of the ongoing global financial crisis 14, exploring structures in firm-level data [18 and assessing risks related to fixed exchange rates 18. Motivated by the experience in these case studies, this paper aims at enhancing the SOTM for it to be applicable in a wider setting.

In this paper, we propose schemes for automated and weighted SOTMs. The first task relates to automating the parametrization with quantitative quality measures. As the SOTM, like the standard SOM, has an aim related to both quantification and topology-preservation accuracy, the parametrization task commonly involves human judgment and manual experimentation. Yet, the goodness measure proposed by Kaski and Lagus [6], which combines quantization and topographic errors, was proposed not only to evaluate the goodness of a SOM, but also to compare a set of SOMs. In this work, we adopt the KaskiLagus measure to the SOTM framework, in order to achieve automated training of the sequence of SOMs, where the parameters for each SOM (with a unique dataset) are derived using the measure. The second task relates to modifying the priority or weighting of data when training a SOTM. While a number of approaches to weighting have been applied in the context of SOMs, the focus herein is on learning that accounts for instance-varying importance. Following the Weighted SOM (WSOM) [17, we augment the neighborhood-functionbased weighting of the batch SOTM (e.g. Voronoi regions) with a user-specified, instance-specific importance weight. This is a broader perspective than incorporating a cost matrix into the objective function of a classifier, which is most often the case in cost-sensitive learning, as the aim of the weighted SOTM is to improve learning from important data with an instance-varying weight. To 
this end, it is not restricted to classification tasks, but rather a feasible alternative for a cost-sensitive, unsupervised version of visual dynamic clustering, as the weight could also represent importance or trustworthiness of an instance for forming clusters. The schemes for automated and weighted SOTMs are illustrated on two real-world datasets: $(i)$ country-level risk indicators to measure the evolution of global imbalances, and $(i i)$ credit applicant data to measure the evolution of firm-level credit risks.

This paper is organized as follows. While Section 2 describes the SOTM and its automation and weighting, Section 3 presents the experiments. Section 4 concludes.

\section{Weighted and automated SOTMs}

This section presents the SOTM, and the automated and weighted training schemes.

\subsection{The SOTM}

The SOTM [16] is an approach for visual dynamic clustering. Yet, it is nothing more than a sequence of one-dimensional SOMs with a short-term memory. The SOMs are trained on data ordered in consequent time units and short-term memory refers to initializations based upon the values of the previous SOM. More formally, for time-coordinates $t$ (where $t=1,2, \ldots, T$ ), the SOTM performs a mapping from the input data space $\Omega(t)$, with a probability density function $p(x, t)$, onto a one-dimensional array $A(t)$ of output units $m_{i}(t)$ (where $i=1,2, \ldots, M)$.

The orientation preservation is performed with the following initializations. The first principal component of Principal Component Analysis is used for initializing $A\left(t_{1}\right)$ to set the orientation of the SOTM. To preserve the orientation between consecutive patterns, the model uses short-term memory. Thus, the orientation of the map is preserved by initializing $\mathrm{A}\left(t_{2,3, \ldots, T}\right)$ with the reference vectors of $A(t-1)$. The training at each $t_{1,2, \ldots, T}$ is iterated for $s_{t}$ training steps, which points to the fact that the number of iterations may vary over $t$ (as also proposed in [16]). For $A\left(t_{1,2, \ldots, T}\right)$, each data point $x_{j}(t) \in \Omega(t)$ (where $j=1,2, \ldots, N(t))$ is compared to reference vectors $m_{i}(t) \in A(t)$ and assigned to its $\mathrm{BMU} m_{b}(t)$ :

$$
\left\|x_{j}(t)-m_{b}\left(t, s_{t}\right)\right\|=\min _{i}\left\|x_{j}(t)-m_{i}\left(t, s_{t}\right)\right\| .
$$

Then, each reference vector $m_{i}(t)$ is adjusted with a batch update:

$$
m_{i}\left(t, s_{t}\right)=\frac{\sum_{j=1}^{N(t)} h_{i b(j)}\left(t, s_{t}\right) x_{j}(t)}{\sum_{j=1}^{N(t)} h_{i b(j)}\left(t, s_{t}\right)},
$$

where index $j$ indicates the input data that belong to unit $b$ and the neighborhood function $h_{i b(j)}\left(t, s_{t}\right) \in(0,1]$ is defined as a Gaussian function 


$$
h_{i b(j)}\left(t, s_{t}\right)=\exp \left(-\frac{\left\|r_{b}(t)-r_{i}(t)\right\|^{2}}{2 \sigma^{2}\left(t, s_{t}\right)}\right),
$$

where $\left\|r_{b}(t)-r_{i}(t)\right\|^{2}$ is the squared Euclidean distance between the coordinates of the reference vectors $m_{b}(t)$ and $m_{i}(t)$ on the one-dimensional array, and $\sigma\left(t, s_{t}\right)$ is the user-specified neighborhood parameter, which may vary over time $t$ and training steps $s_{t}$. Relating to the originally presented SOTM [16], the above introduced notation is similar in nature but allows for training steps $s_{t}$ to be larger than one and vary over time $t$, as well as allows for the radius of the neighborhood $\sigma\left(t, s_{t}\right)$ to change over time $t$ and training steps $s_{t}$. This implies that the training at each point in time may change as per the given needs.

\subsection{Automated SOTMs}

The notion of an automated SOTM relies on having an objective function to minimize. Drawing upon the SOM literature, there has been an extensive discussion of the form and existence of an objective function. Yet, while the literature has provided a wide range of approaches for measuring the quality of a SOM, there is still no commonly accepted objective function for the general case (see, e.g., Yin [22]). This has stimulated the use of a range of quality measures. The most common measures are the standard quantization error $\varepsilon_{q e}$, distortion measure $\varepsilon_{d m}$ [11] and topographic error $\varepsilon_{t e}$ [8], whereas more recent work have extended these measures towards a range of directions. Out of the mentioned measures, the two former ones quantify the quantization quality of the map and the latter one the topological ordering of the map. These have also been proposed as measures for the SOTM:

$$
\begin{gathered}
\varepsilon_{q e}=\frac{1}{T} \sum_{t=1}^{T} \frac{1}{N(t)} \sum_{j=1}^{N(t)}\left\|x_{j}(t)-m_{b(j)}(t)\right\| \\
\varepsilon_{d m}=\frac{1}{T} \sum_{t=1}^{T} \frac{1}{N(t)} \frac{1}{M(t)} \sum_{j=1}^{N(t)} \sum_{i=1}^{M(t)} h_{i b(j)}(t)\left\|x_{j}(t)-m_{b(j)}(t)\right\|, \\
\varepsilon_{t e}=\frac{1}{T} \sum_{t=1}^{T} \frac{1}{N(t)} \sum_{j=1}^{N(t)} u\left(x_{j}(t)\right),
\end{gathered}
$$

where $u\left(x_{j}(t)\right)$ measures the average proportion of $x_{j}(t) \in \Omega(t)$ for which first and second BMU (within $A(t)$ ) are non-adjacent units.

Yet, in order to provide one measure to steer the training of a SOM, the focus herein is on a measure by Kaski and Lagus [6] that combines quantization and topographic errors for evaluating the goodness of a SOM. The Kaski-Lagus measure accounts for the distance between data $x_{j}$ and BMU $m_{b}$ and the shortest path between the coordinates of the first BMU $r_{b}$ and second BMU $r_{b^{\prime}}$. Hence, it measures both the accuracy in representing the data and the continuity of 
the data-to-grid mapping. Formally, the goodness of the map is expressed as follows:

$$
\varepsilon_{k l}=\frac{1}{T} \sum_{t=1}^{T} \frac{1}{N(t)} \sum_{j=1}^{N(t)}\left(\left\|x_{j}(t)-m_{b}(t)\right\|+\min _{p} \sum_{g=1}^{P_{b^{\prime}}\left(x_{j}(t)\right), p}\left\|m_{I_{p}(g-1)}(t)-m_{I_{p}(g)}(t)\right\|\right)
$$

where $b\left(x_{j}(t)\right)$ and $b^{\prime}\left(x_{j}(t)\right)$ represent the index of the first BMU and second BMU and $I_{p}(g)$ denotes the index of the path along the map grid from unit $I_{p}(0)=b\left(x_{j}(t)\right)(\mathrm{BMU})$ to $I_{p}\left(P_{b^{\prime}\left(x_{j}(t)\right), p}\right)=b^{\prime}\left(x_{j}(t)\right)$. For $I_{p}$ to be a path on the grid, the units $I_{p}(g-1)$ and $I_{p}(g)$ need to be neighbors for $g=1, \ldots, P_{b^{\prime}}\left(x_{j}(t)\right), p$. Hence, the first term is the standard quantization error, whereas the second term is a continuity metric measuring whether close points in the mapped space have contiguous BMUs on the map grid. Thus, when adapting the Kaski-Lagus measure to each one-dimensional SOM of the SOTM, we have a single criterion $\varepsilon_{k l}(t)$ to steer the choice of the parameter $\sigma\left(t, s_{t}\right)$ throughout the SOTM training. The implication of a data-driven choice of $\sigma$ is not only an automation of the SOTM training, but also the feature of adjusting the training to the characteristics of the data at each time $t$. Likewise, in order to derive qualities, or properties, of individual SOMs, we can compute each of the quality measures $\varepsilon_{q e}, \varepsilon_{d m}$, and $\varepsilon_{t e}$ at the level of individual one-dimensional SOMs, denoted $\varepsilon_{q e}(t), \varepsilon_{d m}(t)$, and $\varepsilon_{t e}(t)$.

\subsection{Weighted SOTMs}

For a weighted approach, a SOM-based algorithm is suitable, as there has been a number of works on weighted or cost-sensitive learning. In the early days of the SOM, Kohonen [10] introduced weighting of data in order to eliminate border effects on the edges of a SOM. Similarly, in applications of the SOM to image compression, Kim and Ra [7] and Kangas [5] proposed the use of statistical properties of data for weighting. Weighting can also be used to adjust for imbalanced class frequencies and for varying the influence or priority of features in SOM training (e.g., 19]). The weighting in the WSOM [17 is different as it provides means for the user to specify the importance of data for learning, in the unsupervised case for forming clusters. This is also the approach that we propose to be used to weight the SOTM learning.

Following [17, the implemented weighted SOTM learning is based upon the batch version of the SOM algorithm augmented with an instance-specific weight. The weighted counterpart of the SOTM iterates in two steps through $1,2 \ldots, t$, in a similar fashion as the above presented original algorithm. The first step follows the SOTM matching in Eq. 1 by assigning each input data vector $x_{j}(t)$ to its BMU $m_{c}(t)$. In the second step, each prototype $m_{i}(t)$ (where $i=1,2 \ldots, M$ ) is adjusted using a weighted counterpart of the batch update formula. Hence, replacing Eq. 2, the update formula of the weighted SOTM takes the following form: 


$$
m_{i}\left(t, s_{t}\right)=\frac{\sum_{j=1}^{N(t)} w_{j}(t) h_{i b(j)}\left(t, s_{t}\right) x_{j}(t)}{\sum_{j=1}^{N(t)} w_{j}(t) h_{i b(j)}\left(t, s_{t}\right)},
$$

where weight $w_{j}$ is the importance of $x_{j}(t)$ for the learning of patterns. Moreover, as discussed in the beginning of this section, but not further explored in the paper, other types of weighting is obviously possible. For instance, for cases with prior knowledge on the non-uniform importance of individual features, one might weight or scale them to resemble differences in relevance.

\section{Experiments}

This section presents the schemes for automated and weighted SOTMs on two real-world datasets: $(i)$ country-level risk indicators to measure the evolution of global imbalances, and (ii) credit applicant data to measure the evolution of firm-level credit risks.

\subsection{From country risks to global imbalances}

Risk identification and assessment are two key tasks in financial stability surveillance. Recently, in terms of country-level oversight by authorities, the macroprudential approach has emerged as a key means to safeguarding financial stability. Rather than only being concerned with the failure of individual entities, a macroprudential approach takes a holistic view on the financial system with the aim and mandate to ensure system-wide stability. Over the past decades, risk identification has mainly been attempted through predictive modeling, which relies on historical data and conventional statistical methods (see, e.g., [1]) and computational intelligence (see, e.g., 14 and the review therein). Yet, given the changing nature of financial crises, we have not been able to avert from major periods of distress. Rather than mappings from high-dimensional data to a crisis probability through predictive modeling, this motivates exploratory analysis of the high-dimensional temporal and cross-sectional data, not the least for exploring which risk and vulnerability indicators are building up this time. Accordingly, 14 proposed a visual approach to identify the evolution of vulnerabilities and risks through the standard SOTM. The application herein extends this work by taking a truly macroprudential approach to the SOTM. While the SOTM provides an illustration of the risks and vulnerabilities in the cross-section, each entity has been assumed to be of equal system-wide importance. The weighting scheme to the SOTM enables the influence to be specified according to the systemic relevance of each entity.

We apply a SOTM with an automated and weighted training scheme for an abstraction of financial indicators before, during and after the global financial crisis of 2007-2009. As above mentioned, the model herein improves the one in 14, and hence the data used herein are also identical to the data in that work. The dataset includes quarterly input matrices $\Omega(t)$, where rows represent countries and columns binary class variables and 14 country-specific macro-financial 


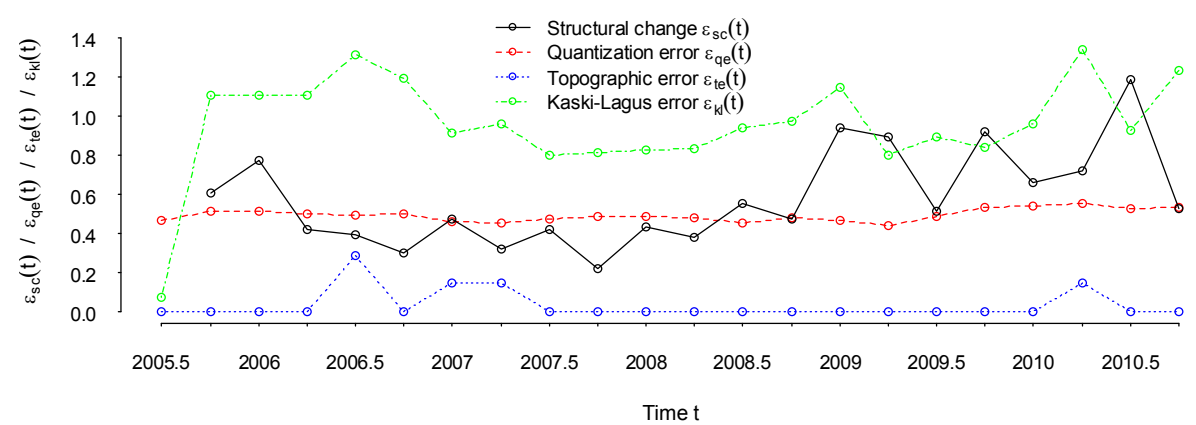

Notes: The errors $\left(\varepsilon_{q e}(t), \varepsilon_{t e}(t)\right.$ and $\left.\varepsilon_{k l}(t)\right)$ are computed for time units $t=1,2, \ldots, T$ and $\varepsilon_{s c}(t)$ for time units $t=2,3, \ldots, T$ for the final model with an $7 \times 22$ array of units and a time-varying $\sigma(t)$.

Figure 1: Quality and property measures of the crisis SOTM.

indicators. The dataset consists of 28 countries, 10 advanced and 18 emerging economies, from 1990:1-2010:3. The class variables represent pre-crisis, crisis, post-crisis and tranquil periods and are objectively identified with a Financial Stress Index (FSI). The FSI defines the crisis events, base upon which we define the rest of the classes. The set of indicators are commonly used measures in the macroprudential literature to capture build-ups of domestic and global vulnerabilities and risks. More specifically, they proxy credit developments and leverage, asset price developments and valuations, as well as more traditional measures of macroeconomic imbalances. Differences in indicators across countries are controlled for by normalizing each input into historic country-specific percentiles. To set the importance of each economy based upon their relevance to the system, we define $w_{j}(t)$ to be the share of stock-market capitalization of country $j$ in period $t$ of the sum of stock-market capitalization in the sample in period $t$. While being an oversimplified measure of an economy's systemic relevance, it is beyond the scope of this paper to create a more advanced measure, which could be metrics from network models, for instance.

The model architecture is set to $7 \times 22$ units, where 7 units represent the cross-sectional dimension and 22 units the time dimension. While the number of units along the quarterly time dimension is set as to span periods before, during and after the crisis that started in 2007 (2005:2-2010:3), the number of units depicting the cross-section at each point in time is determined based upon its descriptive value. Due to the property of a $A(t)$ approximating probability density functions of the data $p(x, t)$, the SOTM, likewise the SOM, is not restricted to treat each unit as an individual cluster. Hence, only the dense locations in the data tend to attract units. As described in Section 2, the Kaski-Lagus error measure $\varepsilon_{k l}(t)$ is used for choosing the final specification of the SOTM. For a SOTM with $7 \times 22$ units, we let the neighborhood radius be a time-varying parameter $\sigma(t)$ to optimize $\varepsilon_{k l}(t)$. Figure 1 shows how $\varepsilon_{k l}(t)$ varies over $t$. Increases in $\varepsilon_{k l}(t)$ can be seen to correlate with increases in $\varepsilon_{q e}(t)$ and/or $\varepsilon_{t e}(t)$. An additional property metric measuring structural change (distance between 


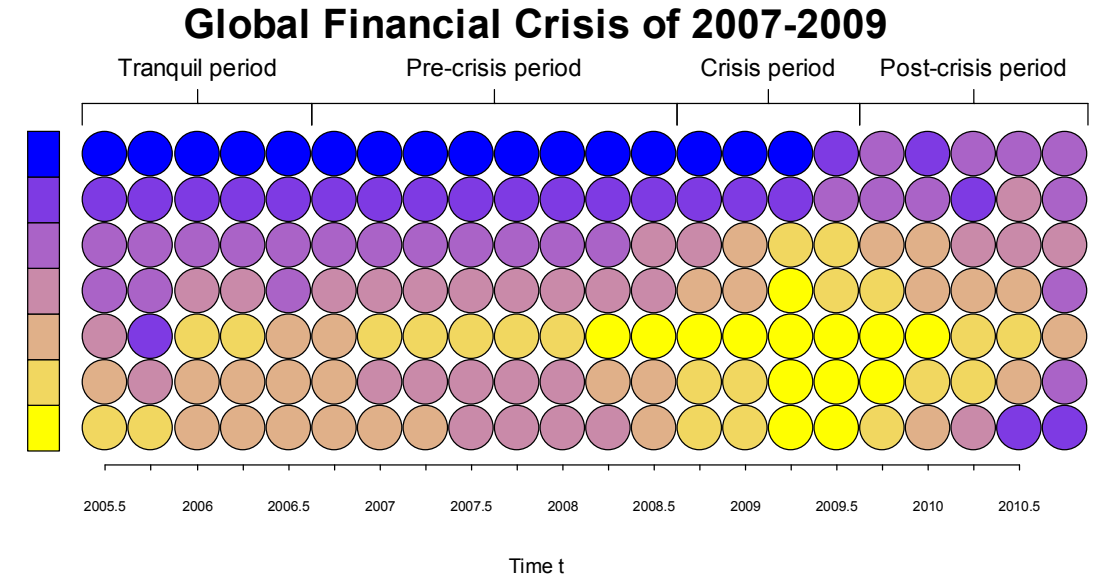

Notes: The figure represents a SOTM of the global financial crisis, where the coloring shows multivariate differences in nodes. Labels above the figure define the classes in data, i.e., the stages of the financial stability cycle.

Figure 2: A SOTM of the global financial crisis.

$A(t)$ and $A(t-1))$ is denoted $\varepsilon_{s c}(t)$, which illustrates that the largest changes in structures occur in the latter part of the sample.

The SOTM is illustrated in Figure 2, where the labels above represent occurrences of the events in the cross-section (averages of the class variables) and the timeline below the figure represents the quarterly time dimension in data. The coloring of the SOTM illustrates the proximity of units as approximated by Sammon's mapping [13. The differences of the units along the vertical direction shows differences in cross-sections, while differences along the horizontal direction shows differences over time. One can observe that in the starting phases of the crisis in 2008, shifts in the color scale towards yellow indicate a start of structural changes. The structural changes reach their peak in 2009, while the structures move back in mid-2010. First, we can observe that the crisis peak is described by the shift towards yellow units. Second, we can observe a post-crisis shift towards a lilac color. Comparing with the observed patterns in [14, the shift towards values of distressed economies (i.e., yellow) is larger when weighting according to stock-market capitalization. This is also natural, as the largest countries were also those suffering most from the crisis.

For a more detailed view of individual risks and vulnerabilities, we can explore the feature planes in Figure 3 . They show for individual inputs (layers of Figure 2 the spread of variable values using a blue scale (with individual scales on the left, in which darker represents higher values). By assessing the feature planes, one can visually both discover spread of risks in the cross-section, and their variation over time.

With no precise empirical treatment of differences between non-weighted and weighted results, the feature planes in Figure 3 generally illustrate expected differences. For instance, the post-crisis period is depicted by a shift in countries 

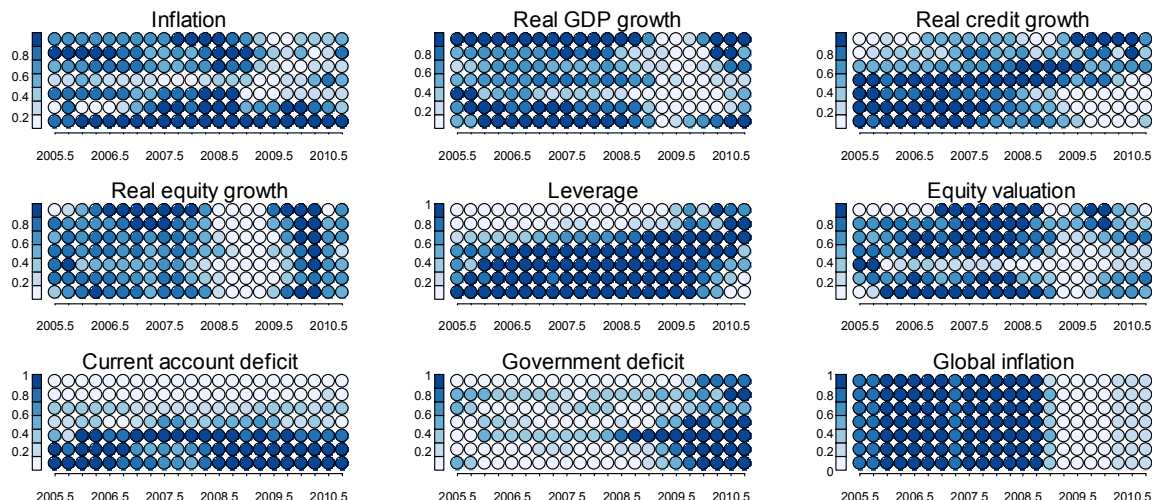

$\begin{array}{llllll}2005.5 & 2006.5 & 2007.5 & 2008.5 & 2009.5 & 2010.5\end{array}$

Current account deficit

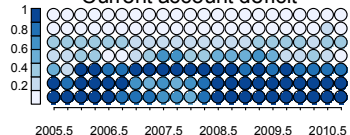

$\begin{array}{llllll}2005.5 & 2006.5 & 2007.5 & 2008.5 & 2009.5 & 2010.5\end{array}$

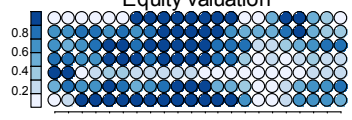

$\begin{array}{llllll}2005.5 & 2006.5 & 2007.5 & 2008.5 & 2009.5 & 2010.5\end{array}$

Global inflation

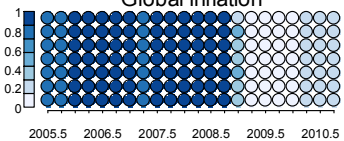

Global real GDP growth

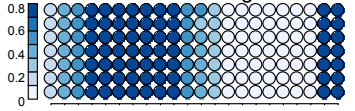

Global real credit growth
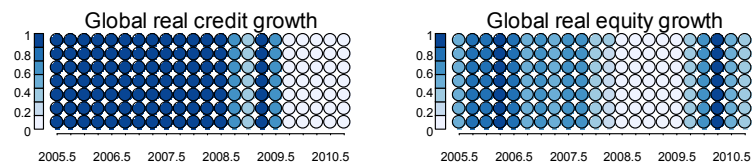

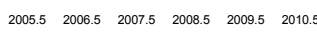

Global leverage

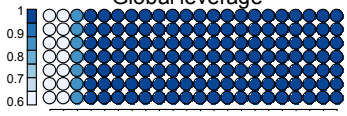

Global equity valuation

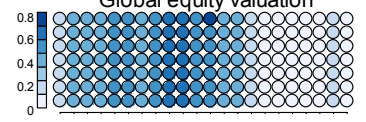

$\begin{array}{llllll}2005.5 & 2006.5 & 2007.5 & 2008.5 & 2009.5 & 2010.5\end{array}$

Crisis periods

Pre-crisis periods
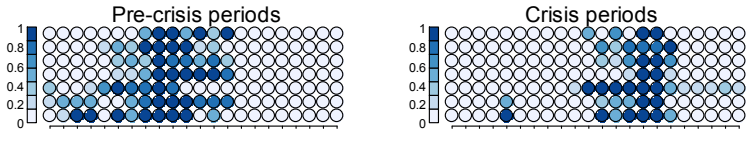

$\begin{array}{llllll}2005.5 & 2006.5 & 2007.5 & 2008.5 & 2009.5 & 2010\end{array}$

$\begin{array}{llllll}2005.5 & 2006.5 & 2007.5 & 2008.5 & 2009.5 & 2010.5\end{array}$

Tranquil periods

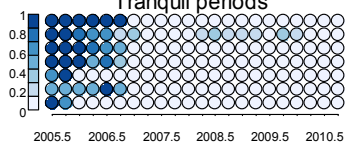

$\begin{array}{llllll}2005.5 & 2006.5 & 2007.5 & 2008.5 & 2009.5 & 2010.5\end{array}$

Post-crisis periods

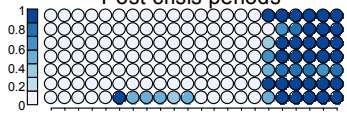

$\begin{array}{llllll}2005.5 & 2006.5 & 2007.5 & 2008.5 & 2009.5 & 2010.5\end{array}$

Notes: The figure shows feature planes for the 14 indicators and the class variables. The feature planes are layers of the SOTM in Figure 2 In the case of binary class variables that take values 1 and 0 , high values represent a high proportion of data in that unit (pre-crisis, crisis, post-crisis or tranquil periods).

Figure 3: Feature planes for the crisis SOTM.

being levered and exhibiting high real credit growth, as well as countries with government deficits. This illustrates that the global financial crisis was mainly driven by imbalances in advanced economies, whereas the countries in the rest of the world are exhibiting risks in credit development. As can be expected, government deficits are shown to have widely increased in the latter part of the analyzed period. In comparison to non-weighted results in [14, we observe larger government deficits, which also holds true with current increases in deficits in countries with large financial systems, such as the United States and the euro area.

\subsection{From credit applicants to firm-level risks}

In firms offering credit to customers, risk management focuses most often on evaluating risks in individual customers. One concern in the evaluation of 


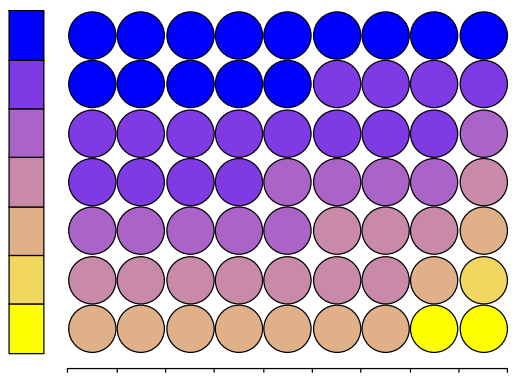

2007.520082008 .520092009 .520102010 .520112011 .52012 Time $\mathrm{t}$

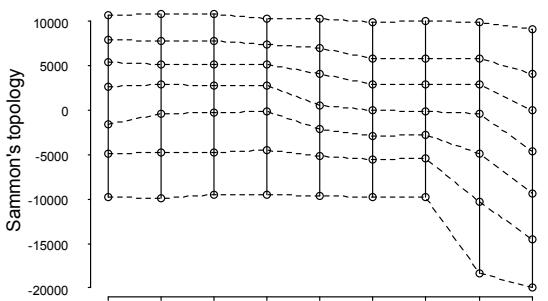

$\begin{array}{lllllllll}2007.5 & 2008 & 2008.5 & 2009 & 2009.5 & 2010 & 2010.5 & 2011 & 2011.5\end{array}$

$$
\text { Time } t
$$

Notes: The figure on the left represents a SOTM of credit applicants in a firm, where the coloring shows multivariate differences in nodes. The right figure shows the underlying Sammon's topology.

Figure 4: A SOTM of credit applicants in firm.

credit risk is, however, the fact that credit scores are non-linearly correlated. When risks build-up over time, and the default probabilities increase, correlations in credit scores tend to increase. Rather than only focusing on individual customers, we take a holistic view on the credit risks building up in a company. In particular, we make use of the SOTM to visualize how the customer portfolio risks are building up over time. The weighting has a natural interpretation also in this case. Each customer contributes to the portfolio risk $\left(w_{j}(t)\right)$ according to the amount of credit that the firm has extended to the debtor at each time point $t$.

In this application, we apply a SOTM with an automated and weighted training scheme for an abstraction of evolving risks in a company providing credit to customers. The dataset used here consists of a full loan database with all debtors for the firm over 4.5 years. The dataset consists of close to 40,000 debtors with a total borrowing of more than $\$ 400$ millions. Model architecture is set to $7 \times 9$ units, where 7 units represent again the cross-sectional dimension, and 9 units represent the time dimension. The number of units along the biannual time dimension is set as to span the available range of data, whereas the number of units describing the cross-section is again determined based upon its descriptive value. As in the previous application, the neighborhood of the radius $\sigma(t)$ is optimized with respect to $\varepsilon_{k l}(t)$.

The credit SOTM is illustrated on the left in Figure 4 As above, the coloring of the SOTM illustrates the proximity of units. Further, the underlying Sammon's topology, which is used for the coloring, is also shown on the right in Figure 4. The figures mainly show that the upper part has been relatively stable, while the lower part illustrates a new, emerging type of credit applicants. The shifts in color on the left in Figure 4 are particularly well communicated with the line graph on the right.

To understand what drives the changes in credit applicants, we can again view the feature planes. In Figure 5 , the feature planes show the spread of individual variables on the credit SOTM. Generally, with no focus on time, the 

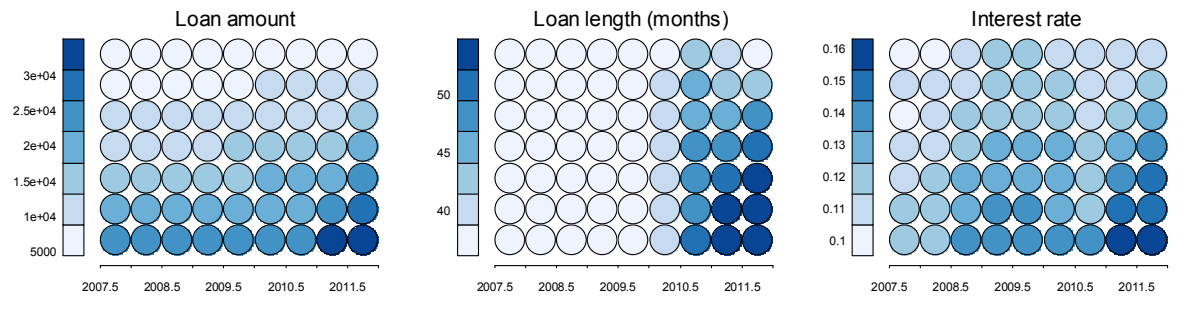

Monthly payments
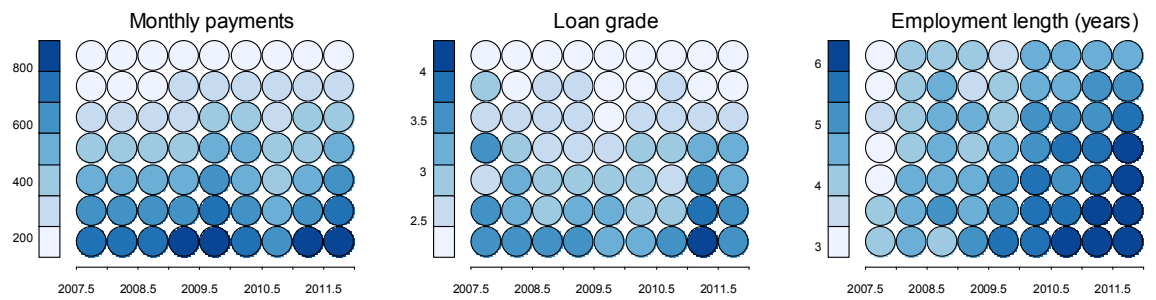

Home, mortgage
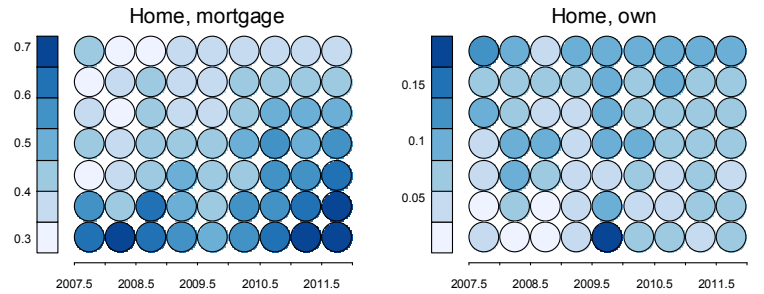

$2007.5 \quad 2008.5 \quad 2009.5 \quad 2010.5 \quad 2011.5$

Debt-to-income ratio

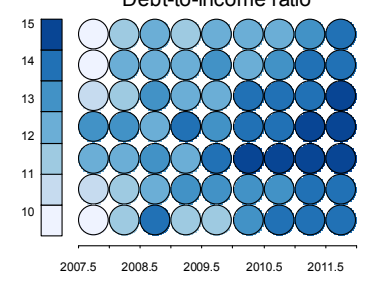

$\begin{array}{lllll}2007.5 & 2008.5 & 2009.5 & 2010.5 & 2011\end{array}$

Lower boundary of FICO
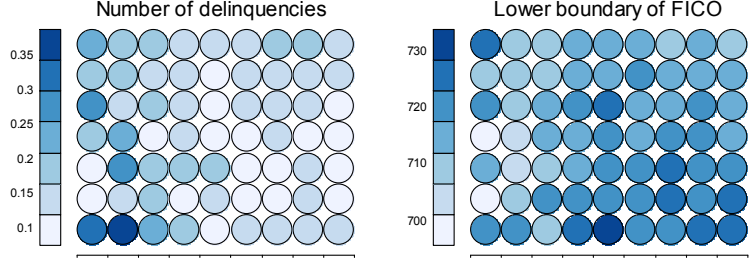

Upper boundary of FICO

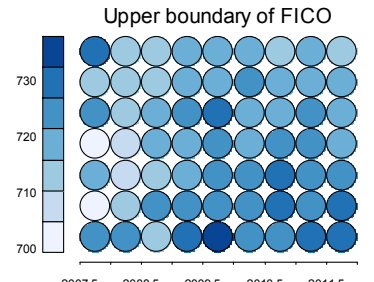

$\begin{array}{lllll}2007.5 & 2008.5 & 2009.5 & 2010.5 & 2011.5\end{array}$

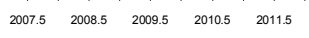

$\begin{array}{lllll}2007.5 & 2008.5 & 2009.5 & 2010.5 & 2011.5\end{array}$

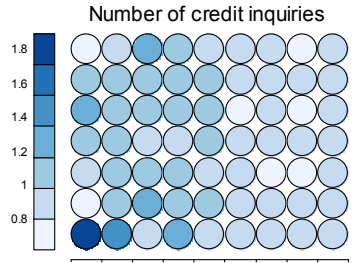

$\begin{array}{lllll}2007.5 & 2008.5 & 2009.5 & 2010.5 & 2011.5\end{array}$

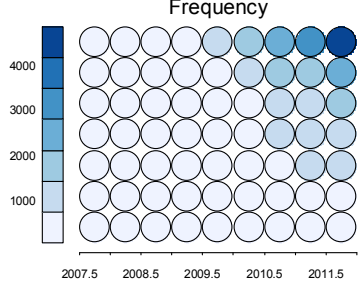

Notes: The figure shows feature planes for the 13 indicators and the frequency of data in each node. The feature planes are layers of the SOTM in Figure 4

Figure 5: Feature planes for the credit SOTM. 
lower part of the map consists of customers with larger and longer loans, with higher interest rates, lower grades and higher monthly payments. These customers have more often a home mortgage, but luckily long historical employment terms. When observing development over time, the feature planes illustrate that loan lengths, sizes and interest rates have increased over time, especially in the lower part of the map. Yet, when combining increases in loans with the income of customers, we can observe that the debt-to-income ratio has not increased most in the lower part of the map, but rather in the center. Moreover, while the lower and central part of the map can be assessed to comprise the customers of lowest grades, the largest increases in frequency occurs in the upper part, which hence can be assessed to indicate growth in healthy loans.

\section{Conclusions}

This paper has proposed two extensions to the SOTM: one for an automated and one for a weighted training scheme. Motivated by the experience in previous work on the SOTM, this paper aims at enhancing it to be applicable in a wider setting. The rationale behind an automated SOTM is not only a data-driven parametrization of the SOTM, but also the feature of adjusting the training to the characteristics of the data at each time step. The implication of a weighting scheme for the SOTM is to improve learning from important data according to an instance-varying weight. Here, importance might refer to a number of notions, such as the certainty or trustworthiness of a data point or the relative relevance of an instance. The schemes for automated and weighted SOTMs have been illustrated on two real-world datasets: $(i)$ country-level risk indicators to measure the evolution of global imbalances, and (ii) credit applicant data to measure the evolution of firm-level credit risks. Among the applications of the SOTM mentioned in the introduction, the weighting scheme would be suitable for illustrating how green customer segments are evolving over time, where the

greenness degree, as estimated from buying behavior, would be the user-specified weight. The general nature of the automation would, on the other hand, be suitable for most cases.

\section{References}

\section{References}

[1] Berg, A., Borensztein, E., Pattillo, C., 2005. Assessing early warning systems: How have they worked in practice? IMF Staff Papers 52(3).

[2] Chakrabarti, D., Kumar, R., Tomkins, A., 2006. Evolutionary clustering. In: Proceedings of the 12th ACM SIGKDD International Conference on Knowledge Discovery and Data Mining (KDD 06). ACM, Philadelphia, PA, pp. 554-560. 
[3] Chakraborty, S., Nagwani, N., 2011. Analysis and study of incremental kmeans clustering algorithm. In: Mantri, A., Saraswati Kendra, S., Kumar, G., Kumar, S. (Eds.), High Performance Architecture and Grid Computing. Communications in Computer and Information Science, Vol. 169. Springer, pp. 338-341.

[4] Holmbom, A., Sarlin, P., Yao, Z., Eklund, T., Back, B., 2013. Visual datadriven profiling of green consumers. In: Proceedings of the International Conference on Information Visualization. IEEE, pp. 291-298.

[5] Kangas, J., 1995. Sample weighting when training self-organizing maps for image compression. In: Proceedings of the 1995 IEEE Workshop on Neural Networks for Signal Processing. pp. 343-350.

[6] Kaski, S., Lagus, K., 1996. Comparing self-organizing maps. In: Proceedings of the International Conference on Artificial Neural Networks (ICANN '96),. Springer, Bochum, Germany,, pp. 809-814.

[7] Kim, K., Ra, J., 1993. Edge preserving vector quantization using selforganizing map based on adaptive learning. In: Proceedings of the International Joint Conference on Neural Networks. IEEE Press, pp. 1219-1222.

[8] Kiviluoto, K., 1996. Topology preservation in self-organizing maps. In: Proceedings of the IEEE International Conference on Artificial Neural Networks. Piscataway, New Jersey, USA, pp. 294-299.

[9] Kohonen, T., 1982. Self-organized formation of topologically correct feature maps. Biological Cybernetics 43, 59-69.

[10] Kohonen, T., 1993. Things you haven't heard about the self-organizing map. In: Proceedings of the International Conference on Neural Networks. pp. 1147-1156.

[11] Lampinen, J., Oja, E., 1992. Clustering properties of hierarchical selforganizing maps. Journal of Mathematical Imaging and Vision 2(2-3), 261272.

[12] Langone, R., Alzate, C., Suykens, J. A. K., 2013. Kernel spectral clustering with memory effect. Physica A: Statistical Mechanics and its Applications 392(10), 2588-2606.

[13] Sammon, J., 1969. A non-linear mapping for data structure analysis. IEEE Transactions on Computers 18(5), 401-409.

[14] Sarlin, P., 2013. Decomposing the global financial crisis: A self-organizing time map. Pattern Recognition Letters 34, 1701-1709.

[15] Sarlin, P., 2013. Replacing the time dimension: A self-organizing time map over any variable. In: Proceedings of the 4th Workshop on New Challenges in Neural Computation (NC2). Machine Learning Reports, Saarbrucken, Germany, pp. 17-24. 
[16] Sarlin, P., 2013. Self-organizing time map: An abstraction of temporal multivariate patterns. Neurocomputing 99(1), 496-508.

[17] Sarlin, P., 2013. A weighted SOM for classifying data with instance-varying importance. International Journal of Machine Learning and Cybernetics, DOI: $10.1007 / \mathrm{s} 13042-013-0175-3$.

[18] Sarlin, P., Yao, Z., 2013. Clustering of the self-organizing time map. Neurocomputing $121,317-327$.

[19] Vesanto, J., Himberg, J., Alhoniemi, E., Parhankangas, J., 1999. SelfOrganizing Map in matlab: the SOM toolbox. In: Proceedings of the Matlab DSP Conference. pp. 35-40.

[20] Yao, Z., Sarlin, P., Eklund, T., Back, B., 2012. Temporal customer segmentation using the self-organizing time map. In: Proceedings of the International Conference on Information Visualisation (iV 12). IEEE Press, Montpellier, France, pp. 234-240.

[21] Yao, Z., Sarlin, P., Eklund, T., Back, B., 2013. Visualizing dynamics in customer behavior with the self-organizing time map. Tech. rep., TUCS Technical Report 1085.

[22] Yin, H., 2008. The self-organizing maps: Background, theories, extensions and applications. In: Fulcher, J., Jain, L. (Eds.), Computational Intelligence: A Compendium. Springer-Verlag, Heidelberg, Germany, pp. 715762 . 